\title{
Spectral Element simulation of rupture dynamics
}

\author{
J.-P. Vilotte ${ }^{1} \&$ G. Festa ${ }^{1}$ \\ ${ }^{1}$ Department of Seismology, Institut de Physique du Globe de Paris, 75252 France
}

\begin{abstract}
Numerical modeling is an important tool, allowing to have access to the different time and space scales carried by the nucleation and propagation of the rupture. In this paper, the spectral element method, which is widely recognized as a powerful tool in regional and global seismology, is applied to the study of the faulting process. Specifically, the importance of the nucleation process in the supershear behavior of inplane cracks is detailed.
\end{abstract}

\section{INTRODUCTION}

Earthquake source dynamics is one of the most important problems in geophysics, providing key elements for the prediction of strong ground motion as well as for the understanding of the physics of earthquake initiation, propagation and healing. Recent studies of rupture processes of selected earthquakes, together with the development of dense digital seismic networks in several seismogenic areas (California, Japan), have shed new lights on the seismic rupture propagation and nucleation. Specifically, they have shown evidence for supershear rupture during the 1999, Izmit, Turkey and the 2001, Kunlunshan, Tibet earthquakes, indentifying the fundamental role that friction and fault geometry play in earthquakes.

In spite of the improvement in computer facilities, rupture propagation modeling remains a difficult problem. Rupture propagation on a major earthquake fault is controlled by the properties of nonregular friction laws that describe the dissipation within the fault interface. Through the initial absolute stress along the fault, friction controls how the rupture initiates, grows from static state to sub or intersonic speeds, and finally stops. Rate- and state-dependent (Dietrich [1]) or simpler slip-weakening (Ida [2]) laws have long been advocated in seismology and both involve an intrinsic finite-length scale which play a major role in the control of the nucleation phase and of the behavior of the rupture front.

An essential requirement to study dynamic faulting is an accurate and robust method for the numerical modeling of earthquake rupturing which allows the resolution of the different time and space scales involved in the nucleation phase, the rupture front propagation and the short wave radiation owing to the heterogeneities and the geometrical complexity of the faults. Two methods have been widely used in seismological applications: the boundary integral equations method (BIEM) and the finite differences (FD). BIEM, pioneered by Das and Aki [3] can handle non planar faults, but it is limited to homogeneous or piecewise homogeneous media, at least in their current implementation. FD, introduced by Madariaga [4] for the study of seismic ruptures, and been since developped by numerous authors, can be used to study rupture propagation in heterogeneous elastic media but with simplified fault geometries.

Spectral element method (SEM) locates at the center of this framework, combining the geometrical flexibility of finite elements with the convergence rate of the spectral methods and it already demonstrated a powerful tool in modeling the seismic wave propagation at regional and global scales (Komatitcsh and Vilotte [5], Capdeville et al. [6]). Taking advantage from the variational approximation, the rupture modeling along non planar flaults through friction laws can be introduced in SEM as an interface condition, in constrast with finite differences, which generally deal with velocity-stress 
staggered grids, where mixed boundary conditions require necessarily some interpolation. Hence, such techniques are expected to require a larger number of points to provide the same spatial resolution as SEM.

\section{RUPTURE MODELING WITH SEM}

From a seismological point of view, the fault is a discontinuity surface of kinematic fields (displacement, velocity and acceleration) embedded in a linear elastic (or viscoelastic) medium. Using a domain decomposition representation, the elastic bulk is separated into two non overlapping regions, independent each of other, whose only interaction is established through the boundary conditions on the common interface. These relationships should account for the continuity of the traction and the frictional laws on the fault surface. On the external surface of the two different domains homogeneous boundary conditions (rigid or free surface) can be imposed instead.

Two terms contribute to the traction on the fault surface: a static contribution, which usually is defined before the rutpuring process and for this reason it is also referred to as initial stress, and the dynamic perturbation, which is generally lower in magnitude and comes from both the elastic bulk and the rupture itself, progessing elsewhere on the fault surface. The total traction, finally, constitutes the kernel of the boundary conditions on the fault plane, that can be summarized in the following.

The contact condition (or Signorini law) requires that either the two sides of the fault do not overlap, or, if opening occurs, both sides act like a free surface. Formally, this yields:

$$
\delta u_{N} \geq 0 ; \quad T_{N} \geq 0 ; \quad \delta u_{N} T_{N}=0
$$

where $\mathbf{T}$ is the traction, $\delta \mathbf{u}$ is the displacement discontinuity across the surface and the index $N$ refers to the normal component of any field.

On the other hand, the frictional condition (Coulomb law) constrains the fault to be at rest until the tangential traction reaches a threshold value, proportional to the normal traction. While sliding, the ratio between tangential and normal traction is controlled by the friction. The formal statement of this condition is:

$$
\Phi\left(\mathbf{T}_{t}, T_{N}\right):=\left(-\left\|\mathbf{T}_{t}\right\|+\mu T_{N}\right) \geq 0 ; \quad \delta \mathbf{v}_{t}=\dot{\gamma} \frac{\mathbf{T}_{t}}{\left\|T_{t}\right\|} ; \quad \dot{\gamma} \leq 0 ; \quad \dot{\gamma} \Phi=0
$$

where $\delta \mathbf{v}$ is the velocity discontinuity, $\mu$ is the frictional coefficient and the index $t$ refers to the fields as projected onto the plane locally tangential to the fault. Through this paper, we adopt a linear slip weakening law, which is widely used in seismological applications (Ida [2]).

Spectral element methods solve the variational statement of the elastodynamic equations by decomposing the elastic domains in hexahedral elements and by approximating the solutions spaces with polynomial subspaces of finite dimension. Integrals involved in the weak formulation are first referred to the single elements and then mapped back to the reference element, that is the cube in 3D. Finally numerical quadrature, applied on the Gauss-Lobatto-Legendre points (which are the same points as used to build up the polynomial basis), transforms the original differential equation into a linear system, whose mass matrix is diagonal. As a consequence, the time evolution of the system can be described through a predictior-multicorrector Newmark scheme, where velocity is recursively updated by using the displacement evaluated at the previous correction, without any matrix inversion. Traction term on the fault is naturally included in the spectral element formulation. Because of the variational feature of the method, the relationship between traction and velocity discontinuity is averaged on the whole fault, as it is seen from the elastic bulk. This smooth condition 


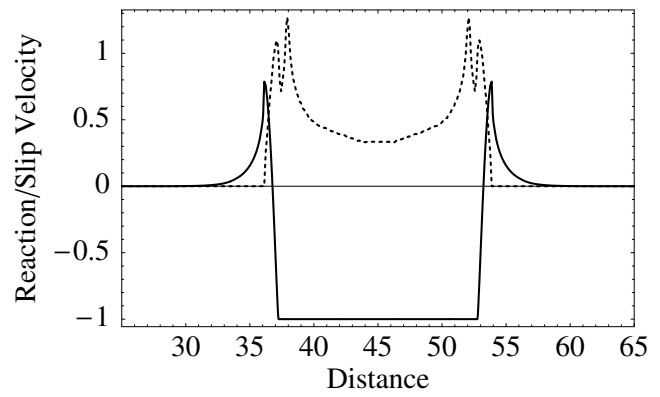

(a)

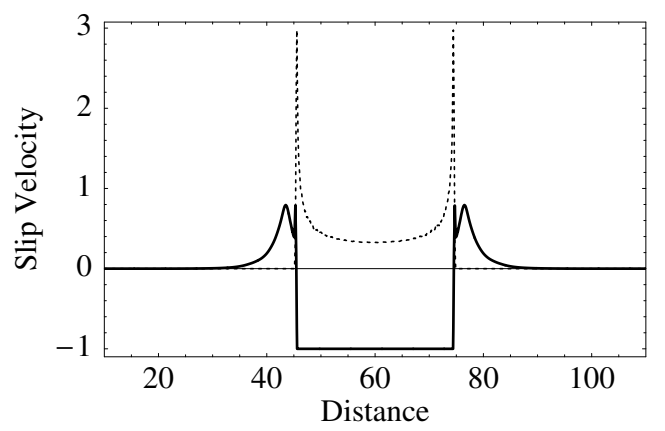

(b)

Figure 1: Slip velocity (dashed curve) and reaction (solid curve) for a supercritic crack (panel a) or a subcritic crack (panel b). In the first case supershear peak grows away from the crack front, without discontinuity. In correspondence, the cohesive zone extends (reaction function bends forward). For a subcritic crack, supershear in carried by the $S$ wave peak in the reaction, allowing the cohesive zone to jump ahead of the front.

should be intersected punctually with the contact and friction laws as described above.

\subsection{High frequency regularization}

Interface conditions are imposed in a strong way on the fault and the discontinuities of the linear slip weakening law generate a signal with a continuous unbounded spectrum. While low frequencies are well propagated by the numerical grid, high frequencies are not scattered away from the fault itself. However, in finite differences grids they are dissipated because of the interpolation. In spectral element meshes instead, they generate spurious modes on the surface interacting in a non linear way with the rupture process. For this reason, a high-frequency regularization of the solutions is strongly required in SEM. This condition can be achieved by embedding the fault in a dispersive anisotropic medium, as obtained by an analytic continuation in the complex space, which has a trasparent behavior for low frequencies and an exponential decay absorption in the high frequency range. The cut-off frequency can be chosen close to the maximum one propagated by the numerical grid according to Courant rule. Attenuation is imposed only along the normal direction and it involves the first layer of elements surrounding the fault itself.

\section{SUPERSHEAR BEHAVIOR OF INPLANE CRACKS}

As an example, we analyze the supershear behavior of the crack as a function of the initial size of crack itself $L$ and the strength parameter $s$ :

$$
s=\frac{\tau_{u}-\tau_{0}}{\tau_{0}-\tau_{f}}
$$

where $\tau_{u}, \tau_{f}$ and $\tau_{0}$ are respectively the strength value, the dynamic stress and the initial traction on the fault. To single out the characteristics of the supershear phase, we take into account the Kostrov's problem of an infinite planar fault embedded in a homogeneous medium, with homogeneous initial conditions and 2D in-plane strain. In this case normal dynamic reaction is zero, because of the symmetry. The dimensions of $L$ should be scaled with respect to the half critical size of the 


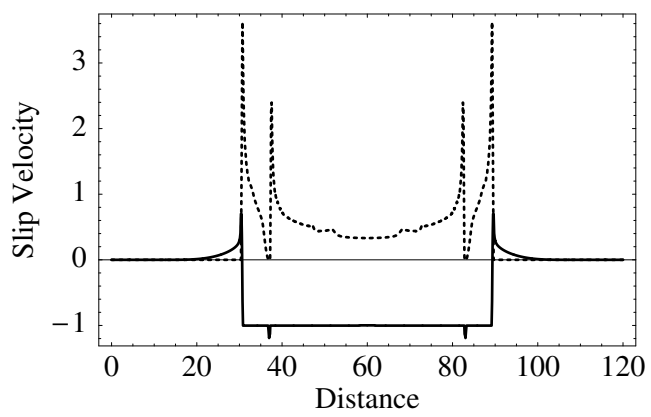

Figure 2: Slip velocity (dashed curve) and reaction (solid curve) during the supershear. While the crack propagates, external peak remains separated from the internal crack front, with a healing phase moving at the $\mathrm{S}$ wave velocity. It is due to a discharge in the reaction, which drops below the dynamic frictional strength.

crack, as defined by Andrews [7].

The rupture process is initiated by raising the initial traction a few percent above the frictional strength in a patch of length $L$, in order to allow the crack to propagate (Andrews [7]).

The most remarkable observation is that supershear occurs in two different ways, depending on both $s$ and $L$ parameters. For large values of $L$ and correspondently small values of $s$, the size of the crack grows at the same time as the nucleation process evolves. At the center of the nucleation zone, the slip rate rises as in a 1D model, with very weak stopping phases coming back into the active zone from the crack tip. When crack speeds up to Rayleigh velocity, slip rate decreases in the central zone to the final constant value, which is close to the $S$ wave velocity (the scaling factor is $\frac{\mu}{\Delta \tau}$ whichisitsel funitary). The supershear peak grows away from the crack front, without changing the shape of the reaction function (fig. 1-a). It progresses initially at about $\sqrt{2} v_{s}$, but it accelerates rapidly toward $\mathrm{P}$ wave velocity, which is never reached, anyway. The supershear peak is always joined to the internal crack front and the peak amplitude becomes more and more important with respect to the internal peak during the propagation of the rupture. We refer this process to as a supercritic crack.

On the other hand, for large $s$ and small $L$ the nucleation phase progress very slowly (subcritic crack). Slip velocity at the center of the initial patch departs very early from the 1D solution, because of stopping phases coming from the boundaries of initial crack. After the nucleation phase, the amplitude decreases to a minimum, lower than the asymptotic value, because most energy is spent to allow the crack to progress. In this case, the crack propagates long time at Rayleigh speed, just to allow the $S$ wave peak in the tangential traction to reach the threshold value (fig. 1-b). Consequently, the cohesive zone jumps ahead of the rupture front, generating a pulse sepated from the internal crack. After an initial adjustment phase, a healing phase propagating at the $\mathrm{S}$ wave speed occurs. The latter is carried by a discharge in the reaction, which drops below the dynamic frictional strength $\tau_{f}$. In this case too, supershear peak accelerates asymptotically toward the $\mathrm{P}$ wave velocity, which is the only admissible speed for this problem(Burridge [8]). 


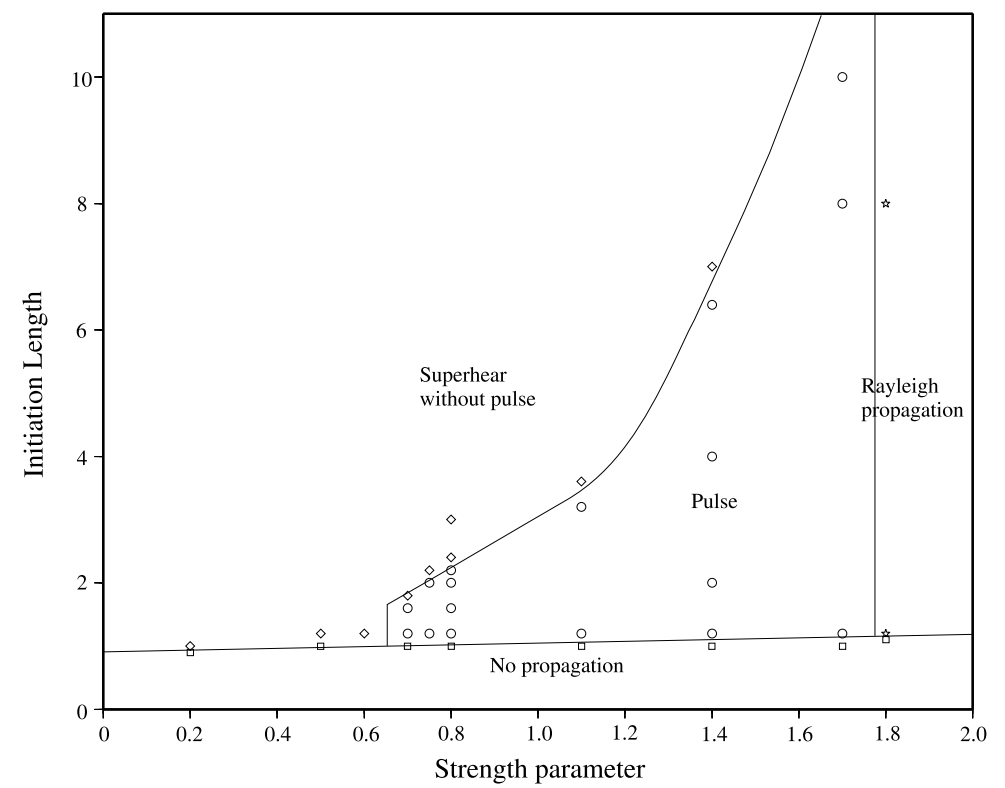

Figure 3: Behavior of crack as a function of the strength parameter $s$ and the size of the initial patch $s$. Four different cases are possible: the crack does not propagate (squares), the crack accelerates moving at supershear speeds with a pulse in the slip velocity ahead of the crack tip (circles), the crack accelerates at supershear speeds without a pulse in advance (parallograms) or finally the crack propagates at Rayleigh wave speed without any supershear phase (stars).

\subsection{Pulse domain}

The importance of different signatures in the supershear phase is not only a numerical results obtained with simplified frictional conditions, but supershear has been always observed in analogical experiments with rock samples (Rosakis et al [9]). Hence, we move to investigate in detail the supershear domain in which the pulse is present, for the same numerical experiment as described in the previous section. In this case, the threshold $\tau_{u}$ and the dynamic value $\tau_{f}$ are assumed constant and changes in the strength parameter $s$ are implied only by the initial stress $\tau_{0}$. Four different have been observed: the crack does not propagate but it stops after a while; the crack moves at the Rayleigh velocity speed; the $S$ wave peak in the reaction reaches the frictional strength allowing the cohesive zone to jump ahead of the crack tip; the crack accelerates at supershear velocities without an advancing pulse. These domains are individuated through experimental points in fig. (3), obtained with numerical simulations. The boundary between the crack propagation and no propagation $L_{n}(s)$ seems to be fit by a straight line with an almost flat slope. In fact, since $\tau_{u}$ and $\tau_{f}$ are fixed, and $\tau_{0}$ is at the threshold in the nucleation zone, the available energy is the same independently of the strength parameter. If we put barriers (an infinite threshold value) at the boundaries of the nucleation zone, the signal will grow indefinitely or will stop only as a function of $L$. If the threshold is finite, sooner or later it will reached, and energy will be subtracted to the nucleation, in order to allow the crack 
to propagate. For two nearby $s$ values, the energy necessary to the crack growth is not significantly different, and therefore, we expect a slow increase of $L_{n}$ as $s$ increases.

The boundary between pulse and no pulse zone $L_{p}(s)$, instead, may not be described by a straight line. In fact, with a weak frictional strength(small values of $s$ ), supershear always occurs without pulse. This limit can be estimated at about $s=0.65$. For $s>0.65$, a pulse occurs in the range $L_{n}(s)<L<L_{p}(s)$. Moreover, this range remains finite for $s=0.65$.

The graph has not many points for large $s$ values, because the computational effort and storage requirements become prohibitive: in this range, larger and larger models should be used to capture the supershear phase. We tested for $s=1.7$, the cases $L=10, L=20$ and $L=40$, and we always observed a pulse carried on by the $\mathrm{S}$ peak. Moreover, for $s>1$, the boundary between the two supershear domains seems to rise as an exponential function.

For $s=1.8$ and reasonable $L$ (just up to $L=20$ ) values, the $\mathrm{S}$ wave peak in the reaction is seen to do not increase its amplitude with time and we are confident that it will not more, because of the self similarity. It agrees with the analytic limit of $s=1.77$ discussed by Burridge [8]. Anyway this condition is obtained for a two value frictional law. For very large $L(L \rightarrow \infty)$, however, the supershear may occur, since a large amount of energy is available before the cohesive zone shrinks.

\section{REFERENCES}

1. Dieterich, J.H., "Modeling of rock friction I: experimental results and constitutive equations", J. Geophys. Res., 84, 2161-2168, 1979.

2. Ida, Y., "Cohesive force across the tip of a longitudinal-shear crack and Griffith's specific surface energy”, J. Geophys. Res., 77, 3796-3805, 1972.

3. Das, S., and Aki, K., "A numerical study of two-dimensional spontaneous rupture propagation”, Geophys. J. R. Astr. Soc., 50, 643-668, 1977.

4. Madariaga, R., "Dynamics of an expanding circular fault", Bull. Seism. Soc. Am., 66, 639666, 1976.

5. Komatitcsh, D., and Vilotte, J.-P., “The Spectral element method: an efficient tool to simulate the seismic response of 2D and 3D geological structures", Bull. Seism. Soc. Am., 88(2), 368-392, 1998.

6. Capdeville, Y., Chaljub, E., Vilotte, J.-P., and Montagner, J.-P., "Coupling the spectral element method with a modal solution for elastic wave propagation in global earth models", Geophys. J. Int., 152, 34-67, 2003.

7. Andrews, D.J., "Dynamic plane-strain shear rupture with a slip-weakening law, calculated by a boundary integral method", Bull. Seism. Soc. Am., 75(1), 1-21, 1985.

8. Burridge, R., "Admissible speeds for plane-strain self-similar shear cracks with friction but lacking cohesion”, Geophys. J. R. Astr. Soc., 35, 439-455, 1973.

9. Rosakis, A.J., Samudrala, O., and Coker, D., "Intersonic shear crack growth along weak planes", Mat. Res. Innovat., 3, 236-246, 2000. 